\title{
QUIVER COEFFICIENTS ARE SCHUBERT STRUCTURE CONSTANTS
}

\author{
Anders Skovsted Buch, Frank Sottile, and Alexander Yong
}

\section{Introduction}

Buch and Fulton established a formula for the cohomology class of a quiver variety [9], which Buch later extended to $K$-theory [5]. The $K$-theory formula expresses a quiver class as an integer linear combination of products of stable Grothendieck polynomials. Quiver coefficients are the coefficients of this linear combination. The quiver coefficients were conjectured to be nonnegative in cohomology and to alternate in sign in $K$-theory. These conjectures were recently proved by Knutson, Miller, and Shimozono [15], Buch [7], and Miller [21]; see also [8].

Buch, Kresch, Tamvakis, and Yong [11, 12] gave combinatorial formulas for the decomposition coefficients expressing a Grothendieck polynomial as an integer linear combination of products of stable Grothendieck polynomials. In particular, it was proved that the decomposition coefficients alternate in sign.

Alternation in sign also occurs in the Schubert calculus of the flag variety; Brion [3] proved that the $K$-theory Schubert structure constants alternate in sign.

We give natural and explicit equalities between the three aforementioned integers. Our argument uses results of Bergeron and Sottile [1] and Lenart, Robinson, and Sottile [20] who earlier established a connection between the decomposition coefficients and the Schubert structure constants. The other main ingredient is the ratio formula of Knutson, Miller, and Shimozono [15], or rather an identity derived from it [7]. We also give a direct argument that the decomposition coefficients have alternating signs, based on Brion's theorem, which then implies that quiver coefficients have alternating signs. A consequence of our theorem is that formulas for the other numbers give formulas for the quiver coefficients; we give examples in the last section.

Received by the editors July 8, 2005.

1991 Mathematics Subject Classification. 05E05, 14M15, 06A07.

Key words and phrases. Quiver coefficient, Grothendieck polynomial, Schubert calculus.

Second author supported in part by NSF CAREER grant DMS-0134860. 


\section{The Main Result}

Quiver coefficients $c_{\underline{\mu}}(r)$ are defined for a set of rank conditions $r=\left\{r_{i, j}\right\}$ for $0 \leq i \leq j \leq n$ and a sequence of partitions $\mu=\left(\mu_{1}, \mu_{2}, \ldots, \mu_{n}\right)$, where $\mu_{i}$ fits in a $r_{i-1} \times r_{i}$ rectangle (for convenience, set $\bar{r}_{i}:=r_{i, i}$ ). The Grothendieck polynomial $\mathfrak{G}_{w}$ for a permutation $w$ represents the class of the structure sheaf of the corresponding Schubert variety in the Grothendieck ring of the flag variety [19]. These form a $\mathbb{Z}$-linear basis for the Grothendieck ring. The integer Schubert structure constants $C_{u, v}^{w}$ are defined by the identity

$$
\mathfrak{G}_{u} \cdot \mathfrak{G}_{v}=\sum_{w} C_{u, v}^{w} \mathfrak{G}_{w}
$$

Brion's theorem [3, Thm. 1] proves that they alternate in sign, $(-1)^{\ell(w u v)} C_{u, v}^{w} \geq$ 0 .

For a partition $\lambda$, let $w(\lambda, k)$ denote the Grassmannian permutation for $\lambda$ with descent at $k$. It is given by $w(\lambda, k)(i)=i+\lambda_{k+1-i}$ for $1 \leq i \leq k$ and $w(\lambda, k)(i)<$ $w(\lambda, k)(i+1)$ for $i \neq k$. The Grothendieck polynomial $\mathfrak{G}_{w(\lambda, k)}\left(y_{1}, y_{2}, \ldots\right)$ is symmetric in the variables $y_{1}, \ldots, y_{k}$ and is independent of $y_{i}$ for $i>k$. Thus we can write $G_{\lambda}\left(y_{1}, \ldots, y_{k}\right)$ for this symmetric Grothendieck polynomial, without ambiguity.

Suppose that $v$ is a permutation whose descents occur at positions in $\left\{r_{0}, r_{0}+\right.$ $\left.r_{1}, \ldots, r_{0}+\cdots+r_{n-1}\right\}$. If $x^{i}=\left(x_{1}^{i}, x_{2}^{i}, \ldots, x_{r_{i}}^{i}\right)$ is a set of $r_{i}$ variables, then the Grothendieck polynomial $\mathfrak{G}_{v}\left(x^{0}, x^{1}, \ldots, x^{n}\right)$ is separately symmetric in each set of variables $x^{i}$ and is independent of $x^{n}$. As the symmetric Grothendieck polynomials $\left\{G_{\lambda}\right\}$ form a basis for all symmetric polynomials, there are integer decomposition coefficients $b_{\underline{\mu}}(v)$ defined by the identity

$$
\mathfrak{G}_{v}\left(x^{0}, x^{1}, \ldots, x^{n}\right)=\sum_{\underline{\mu}} b_{\underline{\mu}}(v) G_{\mu_{1}}\left(x^{0}\right) G_{\mu_{2}}\left(x^{1}\right) \cdots G_{\mu_{n}}\left(x^{n-1}\right) .
$$

Formulas for these coefficients given in [12] show that $(-1)^{\sum\left|\mu_{i}\right|-\ell(v)} b_{\underline{\mu}}(v) \geq 0$. In Remark 3 below, we give a simple geometric argument that accounts for this alternation in sign, via Brion's theorem.

Our main result requires some notation and terminology. For a set of rank conditions $r$, let $d_{i}^{\prime}:=r_{i}+\cdots+r_{n-1}$ and $d_{i}:=d_{i}^{\prime}+r_{n}$. Also let $R_{i}=\left(d_{i+1}\right)^{r_{i-1}}$ be the rectangular partition with $r_{i-1}$ rows and $d_{i+1}$ columns. For a sequence of partitions $\mu=\left(\mu_{1}, \mu_{2}, \ldots, \mu_{n}\right)$ let $\tilde{\mu}_{i}$ be the result of attaching $\mu_{i}$ to the right side of $R_{i}$, and let $\underline{\mu}=\left(\widetilde{\mu}_{1}, \ldots, \widetilde{\mu}_{n}\right)$ denote the sequence of these partitions.

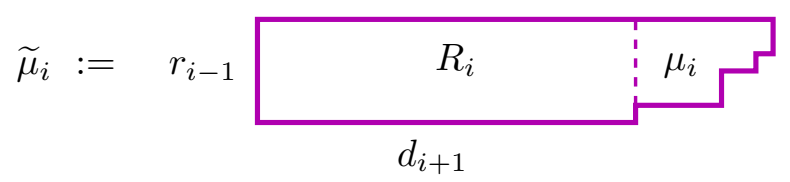

Let $\rho$ be a concatenation of rectangles of sizes $d_{i}^{\prime} \times d_{i}$ for $i=1,2, \ldots, n-1$. This is the shaded part of the partition shown in (3). Finally, we let $\rho(\underline{\mu})$ be the 
partition obtained when $\widetilde{\mu}_{i}$ is placed under the $i$ th rectangle of $\rho$ and the result is concatenated with $\mu_{n}$. This has $d:=d_{0}^{\prime}$ rows and is shown below.

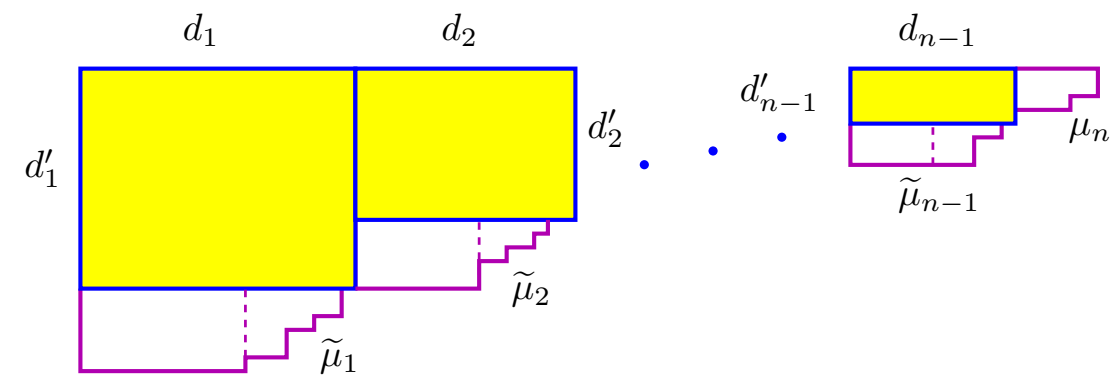

The Zelevinsky permutation $v(r)$ encodes the rank conditions $r$; we refer to [15, Def. 1.7] for the precise definition. We note that the descents of $v(r)$ occur at positions in $\left\{r_{0}, r_{0}+r_{1}, \ldots, r_{0}+\cdots+r_{n-1}\right\}$.

Theorem 1. Let $r$ be a set of rank conditions and $\underline{\mu}=\left(\mu_{1}, \ldots, \mu_{n}\right)$ be a sequence of partitions with $\mu_{i}$ a subset of the $r_{i-1} \times r_{i}$ rectangle. Then the following numbers are equal:

(I) the quiver coefficient $c_{\mu}(r)$;

(II) the decomposition coefficient $b_{\underline{\widetilde{\mu}}}(v(r))$;

(III) the Schubert structure constant $C_{v(r), w(\rho, d)}^{w(\rho(\mu), d)}$.

We follow the notation for quiver coefficients used in [15], which conjugates all partitions compared to the notation used in $[9,5,7]$.

Proof. Zelevinsky [22] showed that a quiver variety for a set $r$ of rank conditions is isomorphic to an open subset of a Schubert variety indexed by the Zelevinsky permutation $v(r)$. Knutson, Miller, and Shimozono used this to prove their ratio formula, which expresses a quiver class as a quotient of two Grothendieck polynomials [15, Thm. 2.7]. We need the following identity which is derived from the ratio formula in $[7, \S 7]$ :

$$
\frac{\mathfrak{G}_{v(r)}\left(x^{0}, x^{1}, \ldots, x^{n}\right)}{\mathfrak{G}_{v(\varepsilon)}\left(x^{0}, x^{1}, \ldots, x^{n}\right)}=\sum_{\underline{\mu}} c_{\underline{\mu}}(r) G_{\mu_{1}}\left(x^{0}\right) G_{\mu_{2}}\left(x^{1}\right) \cdots G_{\mu_{n}}\left(x^{n-1}\right) .
$$

Here $\varepsilon$ denotes the maximal rank conditions given by $\varepsilon_{i j}=\min \left\{r_{i}, \ldots, r_{j}\right\}$. Notice that the cohomology version of (4) follows from Thm. 7.10 and Prop. 7.13 of [15]. The denominator of (4) is the monomial

$$
\mathfrak{G}_{v(\varepsilon)}\left(x^{0}, x^{1}, \ldots, x^{n}\right)=\prod_{i=1}^{n-1} G_{R_{i}}\left(x^{i-1}\right)=\prod_{i=1}^{n-1}\left(x_{1}^{i-1} x_{2}^{i-1} \cdots x_{r_{i-1}}^{i-1}\right)^{d_{i+1}} .
$$

This formula follows from the special form of the permutation $v(\varepsilon)$ (denoted $v$ (Hom) where it was originally defined in [15, Def. 1.7.10]). This permutation is dominant, and thus the Grothendieck polynomial is the monomial associated to the diagram of the permutation; this latter fact can be derived from, e.g., [13]. 
By using that $G_{R_{i}}\left(x^{i-1}\right) G_{\mu_{i}}\left(x^{i-1}\right)=G_{\widetilde{\mu}_{i}}\left(x^{i-1}\right)[6$, Cor. 6.5$]$ we deduce that

$$
\mathfrak{G}_{v(r)}\left(x^{0}, x^{1}, \ldots, x^{n}\right)=\sum_{\underline{\mu}} c_{\underline{\mu}}(r) G_{\widetilde{\mu}_{1}}\left(x^{0}\right) G_{\widetilde{\mu}_{2}}\left(x^{1}\right) \cdots G_{\widetilde{\mu}_{n}}\left(x^{n-1}\right) .
$$

This proves the equivalence of (I) and (II). Now since the last descent of $v(r)$ occurs before position $d$, it follows from [20, Thm. 9.7] that

$$
\mathfrak{G}_{v(r)}\left(x^{0}, x^{1}, \ldots, x^{n}\right)=\sum_{u_{i} \in \mathcal{S}_{d_{i-1}}} C_{v, w(\rho, d)}^{\left(u_{1} \times \cdots \times u_{n}\right) \cdot w(\rho, d)} \mathfrak{G}_{u_{1}}\left(x^{0}\right) \cdots \mathfrak{G}_{u_{n}}\left(x^{n-1}\right),
$$

where $\mathcal{S}_{d_{i-1}}$ is the symmetric group on $d_{i-1}$ elements, and $u_{1} \times \cdots \times u_{n} \in$ $\mathcal{S}_{d_{0}+\cdots+d_{i-1}}$ is the Cartesian product of the permutations $u_{i}$. Because the left hand side of (5) is symmetric in each set of variables $x^{i}$, it follows that any permutation $u_{i}$ which occurs in (5) with a non-zero coefficient must be Grassmannian with descent at position $r_{i-1}$, so $u_{i}=w\left(\lambda_{i}, r_{i-1}\right)$ for a partition $\lambda_{i}$. Furthermore, since $G_{R_{i}}\left(x^{i-1}\right)$ divides the left hand side of (5), each partition $\lambda_{i}$ must have the form $\widetilde{\mu}_{i}$ for a partition $\mu_{i}$. One may check that

$$
\left(w\left(\widetilde{\mu}_{1}, r_{0}\right) \times \cdots \times w\left(\widetilde{\mu}_{n}, r_{n-1}\right)\right) \cdot w(\rho, d)=w(\rho(\underline{\mu}), d) .
$$

Together with (5), this gives

$$
\mathfrak{G}_{v(r)}\left(x^{0}, x^{1}, \ldots, x^{n}\right)=\sum_{\underline{\mu}} C_{v(r), w(\rho, d)}^{w(\rho(\mu), d)} G_{\widetilde{\mu}_{1}}\left(x^{0}\right) G_{\widetilde{\mu}_{2}}\left(x^{1}\right) \cdots G_{\widetilde{\mu}_{n}}\left(x^{n-1}\right) .
$$

This proves the equivalence of (II) with (III).

Example 2. Suppose that $n=3$ and let $r$ be the set of rank conditions

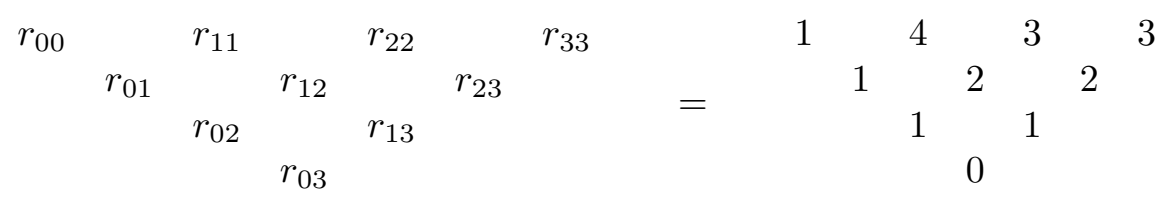

Here, $\left(d_{0}^{\prime}, d_{1}^{\prime}, d_{2}^{\prime}\right)=(8,7,3),\left(d_{0}, d_{1}, d_{2}, d_{3}\right)=(11,10,6,3)$, and the Zelevinsky permutation $v(r)$ is $(7,4,5,8,9,1,2,11,3,6,10)$. The partition $\rho$ is the concatenation of a $7 \times 10$ rectangle with a $3 \times 6$ rectangle, and so equals $(16,16,16,10,10,10,10)$. This is the shaded part of the partition shown in (7).

Let $\underline{\mu}=(\emptyset,(2,1,1),(1))$ be a sequence of partitions. Then $\widetilde{\mu}=((6)$, $(5,4,4, \overline{3}),(1))$ and the partition $\rho(\mu)$ is $(17,16,16,15,14,14,13,6)$, which is illustrated below.

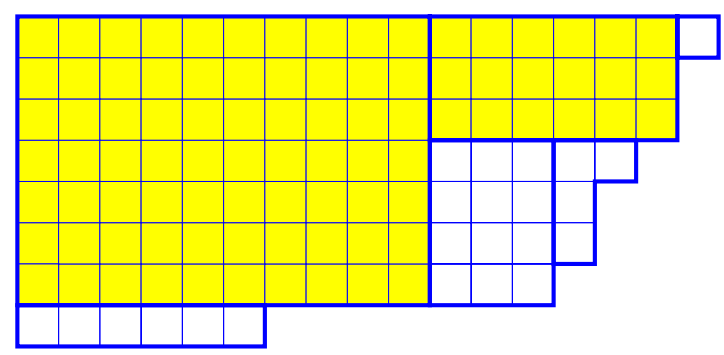


In Example 1 of [4], the quiver coefficient $c_{\underline{\mu}}(r)$ was computed to be 1 . Due to our different conventions, the corresponding term there is written $1 \otimes s_{\square}^{\square} \square_{\square}$, which is indexed by the sequence of partitions $(\emptyset,(3,1),(1))$. Later, we will use formulas from [11] and [17] to calculate that the corresponding decomposition coefficients and Schubert structure constants are both 1 .

Remark 3. The alternating signs of the decomposition coefficients can be explained geometrically using Brion's theorem as follows. Let $v$ be a permutation whose descents occur at positions in $\left\{r_{0}, r_{0}+r_{1}, \ldots, r_{0}+\cdots+r_{n-1}\right\}$. Fix a large integer $N$ and let $Y=\operatorname{Fl}\left(r_{0}, r_{0}+r_{1}, \ldots, r_{0}+\cdots+r_{n-1} ; \mathbb{C}^{n N}\right)$ be the variety of partial flags of the indicated type in $\mathbb{C}^{n N}$. Then $\mathfrak{G}_{v}$ represents the class $\left[\mathcal{O}_{Y_{v}}\right]$ of the structure sheaf of the Schubert variety $Y_{v}$ of $Y[19]$. The product of Grassmannians

$$
X=\operatorname{Gr}\left(r_{0}, N\right) \times \operatorname{Gr}\left(r_{1}, N\right) \times \cdots \times \operatorname{Gr}\left(r_{n-1}, N\right)
$$

can be identified with a subvariety of $Y$ by mapping each point $\left(V_{1}, \ldots, V_{n}\right) \in X$ to the partial flag $V_{1} \subset V_{1} \oplus V_{2} \subset \cdots \subset V_{1} \oplus \cdots \oplus V_{n}$ in $Y$. If we let $x^{i}=\left\{x_{1}^{i}, \ldots, x_{r_{i}}^{i}\right\}$ denote the $K$-theoretic Chern roots of the dual of the tautological subbundle corresponding to the $i$ th factor of $X$, then the specialization $\mathfrak{G}_{v}\left(x^{0}, x^{1}, \ldots, x^{n}\right)$ is obtained by restricting the class $\left[\mathcal{O}_{Y_{v}}\right]$ to the Grothendieck ring of $X$. Expanding this restriction in the basis of Schubert structure sheaves on the flag variety $X$ gives

$$
\mathfrak{G}_{v}\left(x^{0}, x^{1}, \ldots, x^{n}\right)=\left[\mathcal{O}_{X_{v}}\right]=\sum b_{\underline{\mu}}(v) G_{\mu_{1}}\left(x^{0}\right) G_{\mu_{2}}\left(x^{1}\right) \cdots G_{\mu_{n}}\left(x^{n-1}\right) .
$$

When the Schubert variety $Y_{v}$ is in general position, the subvariety $X_{v}:=X \cap Y_{v}$ of $X$ has rational singularities [3, Lemma 2]. By [3, Thm. 1], we have that $(-1)^{\sum\left|\mu_{i}\right|-\ell(v)} b_{\underline{\mu}}(v) \geq 0$, as required. This, together with Theorem 1 proves that the quiver coefficients alternate in sign.

In [1] similar geometry is used to study the restriction of Schubert classes $\left[Y_{v}\right]$ in a full flag manifold $Y$ to products $X$ of flag manifolds embedded in $Y$ in a similar fashion to that given here. There, the subvariety $X_{v}$ is identified as an intersection of Schubert varieties in $Y$. This is used to identify the decomposition coefficients as particular Schubert structure constants, for cohomology. The analogous identification of $K$-theoretic decomposition coefficients with $K$ theoretic Schubert structure constants is accomplished in [20].

\section{Alternative formulas for quiver coefficients}

By Theorem 1, formulas for the decomposition coefficients and Schubert structure constants give alternative formulas for the quiver coefficients. We give two examples of this for the cohomology quiver coefficients (which are indexed by sequences of partitions such that $\sum\left|\mu_{i}\right|$ equals the expected codimension of the quiver variety, see [9]).

The formulas of $[11,12]$ for the decomposition coefficients give formulas for quiver coefficients in cohomology and in $K$-theory. We state this formula for the 
cohomology decomposition coefficients. Suppose that $v$ is a permutation whose descents occur at positions in $\left\{r_{0}, r_{0}+r_{1}, \ldots, r_{0}+\cdots+r_{n-1}\right\}$. The decomposition coefficient $b_{\mu}(v)$ is equal to the number of sequences of semistandard tableaux $\left(T_{1}, \ldots, T_{n}\right)$ such that

(i) The shape of $T_{i}$ is the conjugate (matrix transpose) of the partition $\mu_{i}$,

(ii) The entries of $T_{i}$ are strictly larger than $r_{0}+\cdots+r_{i-2}$, and

(iii) Concatenating the bottom-up, left-to-right column reading words of the tableaux $T_{1}, T_{2}, \ldots, T_{n}$ gives a reduced word for $v$.

The quiver coefficient computed in Example 2 corresponds to the sequence of tableaux

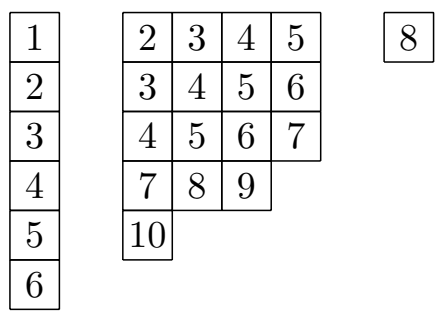

which encodes the following reduced word:

$$
v(r)=s_{6} s_{5} s_{4} s_{3} s_{2} s_{1} s_{10} s_{7} s_{4} s_{3} s_{2} s_{8} s_{5} s_{4} s_{3} s_{9} s_{6} s_{5} s_{4} s_{7} s_{6} s_{5} s_{8} .
$$

Second, Kogan [17] gives a generalization of the Littlewood-Richardson rule for the cohomology Schubert structure constants $C_{v, w(\lambda, d)}^{u}$, when $v$ has no descents after position $d$. We give a mild reformulation of his formula in terms of chains in the Bruhat order from $v$ to $u$ that give valid reading words for tableaux of shape $\lambda$.

A saturated chain $\gamma$ in the $d$-Bruhat order is a sequence of permutations

$$
\gamma: v=v_{0} \longrightarrow v_{1} \longrightarrow v_{2} \longrightarrow \cdots \longrightarrow v_{t}=u,
$$

where $\ell\left(v_{i}\right)=\ell(v)+i$ and $v_{i-1}^{-1} v_{i}$ is a transposition $\left(j_{i}, k_{i}\right)$ with $j_{i} \leq d<k_{i}$ for each $i=1, \ldots, t$. The word of such a chain $\gamma$ is the sequence of integers

$$
v_{1}\left(k_{1}\right), v_{2}\left(k_{2}\right), \ldots, v_{t}\left(k_{t}\right) \text {. }
$$

Kogan's formula [17, Theorem 2.4] asserts that if $v$ has no descents after position $d$, then $C_{v, w(\lambda, d)}^{u}$ is equal to the number of saturated chains in the $d$-Bruhat order from $v$ to $u$ whose word is the left-to-right, bottom-up row reading word of a tableau of shape $\lambda$.

For the quiver coefficient of Example 2, we have $d=8$ and there is exactly one saturated chain in the $d$-Bruhat order that goes from $v(r)$ to $w(\rho(\mu))$ and whose word is a reading word for a semistandard tableau of shape $\rho$.

To describe it, we define a chain in the $d$-Bruhat order to be increasing if its word is an increasing sequence. If there is an increasing chain from $v$ to $u$, then it is unique, the permutation $v u^{-1}$ is the product of disjoint cycles where the numbers decrease in each cycle, and the partition of the numbers by the cycles they lie in is non-crossing [2, p. 655]. The desired chain has length 88 and it is the concatenation of increasing chains of lengths $10,10,10,10,16,16,16$, 
respectively. Below, we display each increasing chain on a separate line. Each product of cycles on a given line is $v_{j} v_{i}^{-1}$, where the increasing subchain for that line is from $v_{i}$ to $v_{j}$.

$$
\begin{aligned}
& (21,20,19,18,17,16,15,14,13,12,11) \\
& (19,18,17,16,15,14,13,12,11,10,9) \\
& (18,17,16,15,14,13,12,11,10,9,8) \\
& (16,15,14,13,12,11,10,9,8,6,5) \\
& (25,24,23,22,21)(20,19)(17,16)(15,14,13,12,11,10,9,8,6,5,4) \\
& (23,22,21,19,16,14,13,12,11,10,9,8,6,5,4,3,3,2) \\
& (22,21,19,16,14,13,12,11,10,9,8,6,5,4,3,2,1)
\end{aligned}
$$

This chain corresponds to the following semistandard tableau of shape $\rho$.

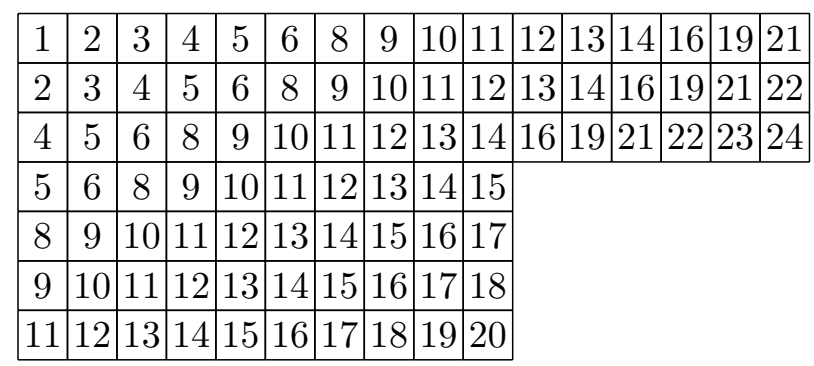

Remark 4. It would be interesting to give bijections between the formulas discussed here for the quiver coefficients (in cohomology) with those given in [15].

Remark 5. Since this paper was submitted, further formulas relevant to quiver coefficients have been discovered, we refer the reader to $[10,16]$ for details.

\section{References}

1. N. Bergeron and F. Sottile, Schubert polynomials, the Bruhat order, and the geometry of flag manifolds, Duke Math. J. 95 (1998), no. 2, 373-423.

2. _ Skew Schubert functions and the Pieri formula for flag manifolds, Trans. Amer. Math. Soc. 354 (2002), no. 2, 651-673 (electronic).

3. M. Brion, Positivity in the Grothendieck group of complex flag varieties, J. Algebra 258 (2002), no. 1, 137-159.

4. A. S. Buch, On a conjectured formula for quiver varieties, J. Algebraic Combin. 13 (2001), no. $2,151-172$.

5. __ Grothendieck classes of quiver varieties, Duke Math. J. 115 (2002), no. 1, 75-103.

6. A Littlewood-Richardson rule for the K-theory of Grassmannians, Acta Math. 189 (2002), no. 1, 37-78.

7. _ Alternating signs of quiver coefficients, J. Amer. Math. Soc. 18 (2005), 217-237.

8. A. S. Buch, L. Fehér and R. Rimányi, Positivity of quiver coefficients through Thom polynomials, to appear in Adv. Math.

9. A. S. Buch and W. Fulton, Chern class formulas for quiver varieties, Invent. Math. 135 (1999), no. 3, 665-687.

10. A. S. Buch, A. Kresch, M. Shimozono, H. Tamvakis and A. Yong, Stable Grothendieck polynomials and $K$-theoretic factor sequences.

11. A. S. Buch, A. Kresch, H. Tamvakis, and A. Yong, Schubert polynomials and quiver formulas, Duke Math. J. 122 (2004), no. 1, 125-143. 
12. Grothendieck polynomials and quiver formulas, to appear in Amer. J. Math.

13. S. Fomin and A. N. Kirillov, Grothendieck polynomials and the Yang-Baxter equation, Proceedings of the 6th Inter. Conf. on Formal Power Series and Algebraic Combinatorics, DIMACS (1994), 183-190.

14. - The Yang-Baxter equation, symmetric functions, and Schubert polynomials, Proceedings of the 5th Conference on Formal Power Series and Algebraic Combinatorics (Florence, 1993), Discrete Math. 153 (1996), no. 1-3, 123-143.

15. A. Knutson, E. Miller and M. Shimozono, Four positive formulae for type A quiver polynomials. Preprint 2003.

16. A. Knutson and A. Yong, A formula for K-theory truncation Schubert calculus, Int. Math. Res. Not. (2004), no. 70, 3741-3756.

17. M. Kogan, RC-graphs and a generalized Littlewood-Richardson rule, Internat. Math. Res. Notices (2001), no. 15, 765-782.

18. A. Lascoux and M.-P. Schützenberger, Polynômes de Schubert, C. R. Acad. Sci. Paris Sér. I Math. 294 (1982), no. 13, 447-450.

19. __ Structure de Hopf de l'anneau de cohomologie et de l'anneau de Grothendieck d'une variété de drapeaux, C.R. Acad. Sci. Paris Sér. I Math. 295 (1982), no. 11, 629-633.

20. C. Lenart, S. Robinson and F. Sottile, Grothendieck polynomials via permutation patterns and chains in the Bruhat order, 2003.

21. E. Miller, Alternating formulae for $K$-theoretic quiver polynomials, to appear in Duke Math. J., 2004.

22. A. V. Zelevinskiı̌, Two remarks on graded nilpotent classes, Uspekhi Mat. Nauk 40 (1985), no. 1 (241), 199-200.

Matematisk Institut, Aarhus Universitet, Ny Munkegade, 8000 Arhus C, Denmark E-mail address: abuch@imf.au.dk

Department of Mathematics, Texas A\& M University, College Station, TX, 77843

E-mail address: sottile@math.tamu.edu

Department of Mathematics, University of California, Berkeley, CA, 94720

E-mail address: ayong@math.berkeley.edu 